\title{
An Effective Progressive Image Transmission using Superpixel based Saliency Detection and Modified SPIHT Compression Algorithm
}

\author{
Ravikiran H. K., Jayanth J
}

\begin{abstract}
A new progressive image transmission system was proposed in this research paper for effective usage of communication bandwidth. At first, the superpixel based saliency detection method was used for segmenting the foreground region from the background region, because it gives more saliency information of an image with the benefit of color contrast. Then, Integer Wavelet Transform (IWT) was applied in the foreground image, which delivers $A$ good quality of the image and also the compression ratio of the image was decent. Additionally, optimized neural network and modified Set Partitioned in Hierarchical Tree (SPIHT) algorithm were applied in the background image that delivers good rate distortion properties in the noise free environment and also enhances the image visual experience. In modified SPIHT, the sub-tree roots were not excluded that helps to encode and quantize the wavelet coefficients effectively. Also, it delivers more information to the image edges that effectively improves the subjective visual experience. Experiment report showed that the proposed work enhanced the Peak Signal to Noise Ratio (PSNR) upto 5dB compared to the existing work.
\end{abstract}

Index Terms: Integer wavelet transform, modified set partitioned in hierarchical tree, neural network, progressive image transmission, and superpixel based saliency detection.

\section{INTRODUCTION}

In recent decades, progressive image transmission plays a vital role in transmitting image over internet channels. The main goal of progressive transmission of an image allows the receiver to recognize the relevant features in an image as quickly as possible at minimum cost [1]. The progressive transmission is achieved by transmitting the low-resolution approximation of an image, and then the further information is transmitted based on the user request. So, the user can easily decide whether the image transmission needs to be continued or not. As a result, the receiver gains more control over the final image quality and the storage space required for the image is also reduced [2-4]. Currently, the progressive

Revised Manuscript Received on December 30, 2019.

* Correspondence Author

Ravikiran H.K.*, Department of Electronics and Communication, GSSS Institute of Engineering and Technology for Women, Affiliated to Visvesvaraya technological university, Mysuru, India,

Dr. Jayanth J, Department of Electronics and Communication, GSSS Institute of Engineering and Technology for Women, Affiliated to Visvesvaraya technological university, Mysuru, India,

(C) The Authors. Published by Blue Eyes Intelligence Engineering and Sciences Publication (BEIESP). This is an open access article under the CC BY-NC-ND license (http://creativecommons.org/licenses/by-nc-nd/4.0/) image transmission is applied in several real-time applications such as medical, teleconferencing, remote image database access, and wildlife monitoring, etc. [5]. The existing progressive image transmission approaches adopted implicitly or explicitly, and the minimal distortion principle is used to decide the importance. In addition, the rate distortion performance is the most important feature for numerous progressive image transmission methods [6-8].

In progressive image transmission methods, it is essential that the initial image quality should be good, so the user can take the decision easily [9-10]. The aim of this research work is to propose a new progressive image transmission system for attaining good quality of images at the early stage with low bit rate. In this paper, the proposed progressive image transmission system is tested on wild life and medical images. Initially, superpixel based saliency detection method is applied on the input images to segment the foreground region from the background region. Then, IWT is applied in the foreground image for converting the image into transform domain that allows a fully in-place calculation, so there is no need of temporary memory. In addition, optimized neural network and modified SPHIT algorithm are used in the background image for compression and decompression process. The proposed algorithms deliver excellent rate distortion characteristics in the noise free environment and also improves the image visual experience. At last, the proposed work performance is related with the prior works by means of PSNR, Structural Similarity Index (SSIM), compression ratio, and Mean Square Error (MSE).

This research paper is written as follows. Some research papers in the progressive image transmission are surveyed in section II. Detailed explanation about the proposed work is specified in section III. Section IV presents experimental results of the proposed method. Conclusion is done in section $\mathrm{V}$.

\section{LITERATURE WORKS}

Currently, many research works are performed in a progressive image transmission. In this literature survey section, description of some crucial contributions to the existing literatures are presented. L. Liu et al. [11] developed a new progressive secret image transmitting scheme with flexible size shadows. Initially, the secret image was measured by using compressed sensing, and then the measured values were subdivided into $n$ shadows by utilizing Shamir's threshold approach.

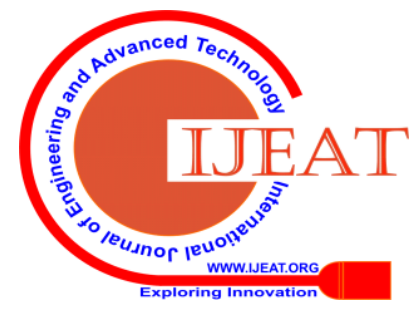


The secret image was reconstructed at the receiver end if any shadows were obtained. Because compressed sensing reconstruction quality was adaptive to the number of measurements, the developed transmitting approach attained flexible shadow size and the property of progressive transmission and error resilient. The simulation outcome shows that the developed scheme attained good performance in view of the reconstructed image. In a few cases, the developed scheme has a bit synchronization property that leads to misinterpretation in the decoding side.

W. Feng et al. [12] presented a new saliency detection scheme on the basis of histogram contrast algorithm for wild animal monitoring. The present research work mainly concentrated on investigating the wildlife images with high non-uniform illumination features, and complex background. At first, structure extraction approach was accomplished for smoothing the image textures based on image total variation. Then, canny edge detection and hanning window were utilized to extract the saliency target edge information. In this literature, the real-time wild animal images were used to validate the efficiency of the developed algorithm. Related to other existing algorithms, the developed algorithm attained better results in light of f-measure, precision, and recall. The developed saliency detection scheme works on the basis of an adaptive pattern table that ultimately requires good quality images.

N. Jiang et al. [13] developed a new Multi Transmission Optimization method (MTO) for medical images in order to investigate the visual content of the images based on the features of mobile telemedicine system. In this work, two enabling approaches (adaptive Representative Image Blocks (RIBs) replicas selection and Non-medically useful area Image Blocks (NIBs) grouping method) were developed for better MTO processing. At first, the correlation between the transmission medical images was explored and then the optimal pixel resolution of RIBs was derived on the basis of current network bandwidth. Besides, the candidates of RIBs and NIBs were transmitted to the receiver end based on the transmission priorities. At last, the image blocks were reconstructed at the receiver end for dissimilar users. Experimental outcome shows that the developed system significantly diminishes the response rime by minimizing the network communication. The major disadvantage in the MTO processing was it highly prone to transmission noise.

H. Kim et al. [14] designed an effective joint source channel coding approach for block fading channel with distributed spatial diversity. The developed method effectively maximizes the source throughput by considering a progressive image coder with constraint transmission bandwidth. In this literature paper, simple approximation formula was applied for identifying an approximation of mutual information outage probability. Then, derive the source throughput expression utilizing progressive source property and mutual information outage probability. The numerical result shows that the developed approach attained better performance in terms of signal to noise ratio. The major drawback in the developed approach was high transmission load.

W. Feng et al. [15] developed a hierarchical coding progressive transmission approach for transmitting the both saliency object region and background region with dissimilar coding priorities and strategies. Initially, convolutional neural network was used to detect the saliency object region by obtaining the mask on wildlife images. Besides, SPIHT coding was utilized to transmit the saliency images that ensured the accuracy of transmission. Then, the background region was transmitted by applying an embedded zerotree wavelets to enhance the transmission efficiency. In this literature, the wildlife animal images were used to verify the effectiveness of the developed progressive transmission approach. From the experimental result, the developed approach achieved a better result related to the existing approaches in light of PSNR, and SSIM. Besides, the developed technique was quite complicated to implement in real-time applications.

To address the above specified concerns, a new progressive image transmission system is proposed to achieve a high quality version of the original image from the minimum amount of data.

\section{PROPOSED TRANSMISSION SYSTEM}

In recent periods, the digital imagining equipment's generates a huge volume of data in a database. Hence, the transmission of high resolution images over a low speed channel will cause a serious delay problem. To address this concern, progressive image transmission system is introduced, which compresses and transmits the coded bit streams under certain constraints.

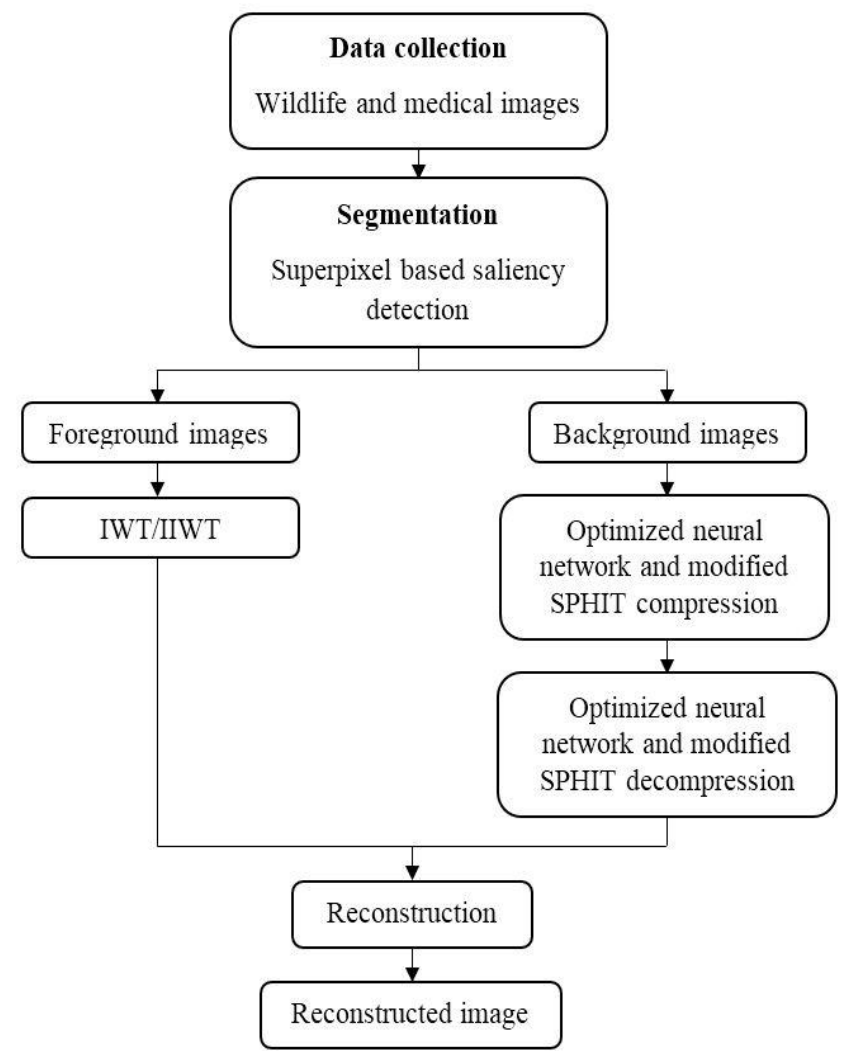

Fig. 1. Block diagram of proposed system

The progressive image transmission system subdivides the transmission process into number of stages and progressively refines the quality of the reconstructed image [16].

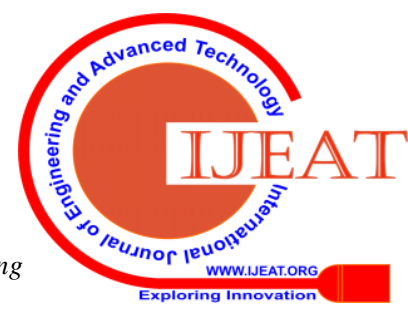


In this research, a new progressive image transmission system is proposed to enhance the perceptual quality of progressively transmitted images. The working procedure of the proposed system is stated in Fig. 1, and the detailed explanation about the proposed transmission system is represented in Fig. 1.

\section{A.Data collection}

For progressive image transmission, the images are collected from DICOM dataset and field-captured wildlife images. The DICOM dataset is almost comprises of $3000+$ T1 weighted contrast enhanced brain images. Usually, it is necessary to select appropriate images, otherwise the intended operation may not be achievable. The sample collected images are represented in Fig. 2.
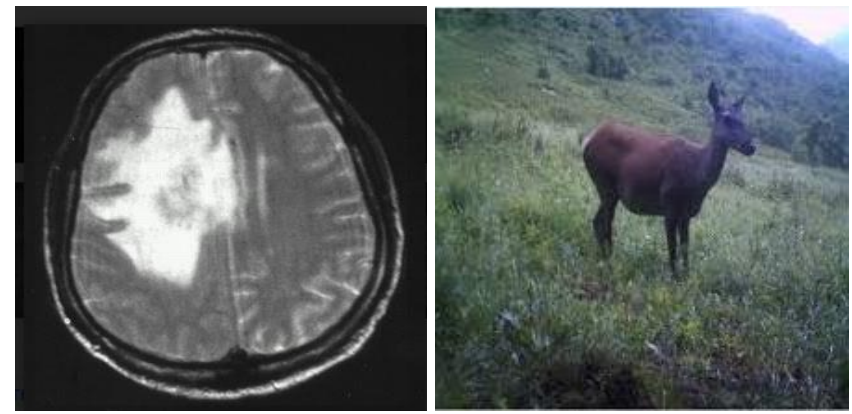

Fig. 2. Sample collected images

\section{B. Superpixel based saliency detection}

After data collection, superpixel based saliency detection is used to segment the foreground region and background region from the images. The proposed saliency detection model contains three stages such as superpixel segmentationadaptive color histogram, inter superpixel similarity, and superpixel saliency [17]. The detailed explanation about the proposed saliency detection model is stated below.

\section{a. Image simplification}

In this segment, superpixel segmentation is carried-out to convert the collected image into lab color space. The proposed superpixel segmentation model works on the basis of linear iterative clustering for subdividing the image into a number of superpixels that generally have a compact and regular shape with better boundary adherence. The size of the superpixel is set to $\sqrt{N / 200}$, where $N$ is represented as number of image pixels, and the number of generated superpixel is 200, which is adequate to preserve the dissimilar boundaries. In adaptive color quantization, each color channel is quantized into $q$ bins for generating the image histogram $H_{0}$. Then, the quantized color of each bin $q c_{k}$ is determined as the mean color of image pixels that falls into the $k^{\text {th }}$ bin, and the high probability bins $m$ have $\alpha, N$ pixels, which is selected as the representative colors. Finally, the remaining bins are merged into one and then the quantized color of all the bins are updated for generating a color quantization table $Q$ with $m$ entries.

\section{b. Inter superpixel similarity}

The superpixel level histogram $H_{x}$ is determined and simplified based on $Q$ by using all pixels in every superpixel $S P_{x}(x=1, \ldots ., n)$, and the normalized superpixel level histogram is mathematically denoted in Eq. (1).

$\sum_{k=1}^{m} H_{x}(k)=1$

Meanwhile, the inter superpixel similarity between every pair of superpixels $S P_{x}$ and $S P_{y}$ is mathematically defined in the Eqs. (2), (3), and (4).

$\operatorname{Sim}(x, y)=\operatorname{Sim}_{\text {spatial }}(x, y) \times \operatorname{Sim}_{\text {color }}(x, y)$

Where,

$\operatorname{Sim}_{\text {color }}(x, y)=\sum_{k=1}^{m} \min \left\{H_{x}(k), H_{y}(k)\right\}$

(3)

$\operatorname{Sim}_{\text {spatial }}(x, y)=1-\frac{\left|\mu_{x}-\mu_{y}\right|}{d}$

Where, $\mu$ is indicated as spatial center position of $S P$, and $d$ is stated as diagonal length of the image.

\section{c. Superpixel saliency}

In most of the natural images, the background superpixels shows color contrast with salient object superpixels and also the spatial distribution of background superpixels are thinner than salient object superpixels that usually scatter over the whole images. On the basis of these observations, the Spatial Sparsity (SS) and Global Contrast (GC) of superpixels are calculated to measure the superpixels saliency. The GC of $S P_{x}$ is determined by using the weighted color differences of other superpixels, as mentioned in Eq. (5).

$G C(x)=\sum_{y=1}^{n} W(x, y) \times\left\|m c_{x}-m c_{y}\right\|$

Where, $m c_{x}$ is stated as mean color of $S P_{x}$. The weight $W(x, y)$ is determined by assuming the factors of spatial similarity and superpixel area, which is mathematically stated in Eq. (6).

$W(x, y)=\left|S P_{y}\right| \times \operatorname{Sim}_{\text {spatial }}(x, y)$

Where, $\left|S P_{y}\right|$ is indicated as number of pixels in the superpixel. In addition, the normalized GC measure of $S P_{x}$ is determined by using the Eq. (7).

Normalized $G C(x)=\frac{G C(x)-G C_{\min }}{G C_{\max }-G C_{\min }}$

Where, $G C_{\min }$ and $G C_{\max }$ are stated as minimum and maximum value in the GC measures of all superpixels. For every superpixel $S P_{x}$, the spatial spread color distribution is represented in Eq. (8).

Published By: 
$S S(x)=\frac{\sum_{y=1}^{n} \operatorname{Sim}(x, y) \times D(y)}{\sum_{y=1}^{n} \operatorname{Sim}(x, y)}$

Where, $D(y)$ is denoted as Euclidean spatial distance from the center position of $S P_{y}$. Besides, the inverse normalization operation is carried-out on the spatial spread measures for obtaining the normalized spatial spread measures for every superpixels that is mathematically represented in Eq. (9).

Normalized $S S(x)=\frac{S S(x)-S S_{\max }}{S S_{\min }-S S_{\max }}$

Where, $S S_{\min }$ and $S S_{\max }$ are indicated as minimum and maximum spatial spread measures of all superpixels. Then, the inter superpixel similarity measures are exploited for refining the SS and GC measures. The refined SS and GC measures are mathematically stated in the Eqs. (10) and (11).

$\operatorname{RSS}(x)=\frac{\sum_{y=1}^{n} \operatorname{Sim}(x, y) \times \text { normalized } S S(y)}{\sum_{y=1}^{n} \operatorname{Sim}(x, y)}$

$R G C(x)=\frac{\sum_{y=1}^{n} \operatorname{Sim}(x, y) \times \text { normalized } G C(y)}{\sum_{y=1}^{n} \operatorname{Sim}(x, y)}$

Where, RSS is indicated as refined spatial sparsity and $R G C$ is stated as refined global contrast. At last, the saliency measure of each superpixel is calculated by performing superpixel wise multiplication that is represented in Eq. (12). The saliency detected images are graphically denoted in Fig. 3.

$$
\operatorname{Sal}(x)=R G C(x) \times R S S(x)
$$

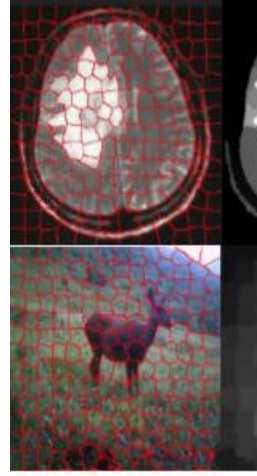

(a)

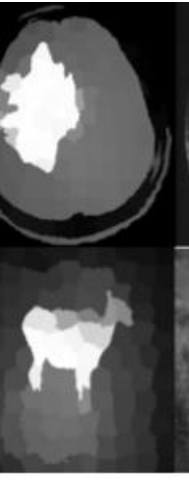

(b)

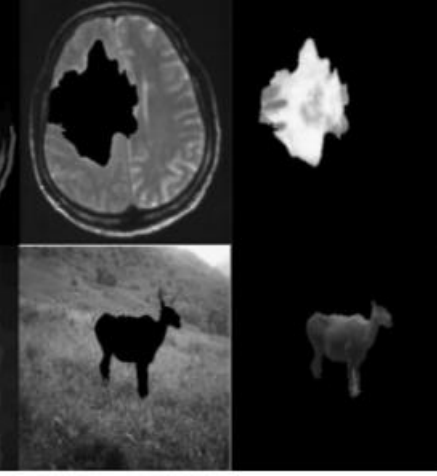

(c)

(d)
Fig. 3. a) Superpixel segmented image, b) saliency detected image, c) background image, d) foreground image

\section{C.Integer wavelet transform}

After performing saliency detection, IWT is applied in the foreground image to convert the image into the transform domain [18]. The wavelet transform is one of the popular approaches used in multi resolution image analysis, especially progressive image transmission. The IWT decomposes the image using detailed and approximate analysis by separating the frequencies into high and low frequencies. Generally, the IWT approach comprises of four sub-band levels such as High-High, High-Low, Low-Low, and Low-High. In this research article, Low-Low sub-band is considered for image transformation, because it appears closely related to the original foreground images. The IWT coefficients are mathematically denoted in Eqs. (13), (14), (15), and (16).

$L L_{x, y}=\left|\frac{\left(O_{2 x, 2 y}+O_{2 x+1,2 y}\right)}{2}\right|$

$H L_{x, y}=O_{2 x+1,2 x^{-}}-O_{2 x, 2 y}$

(14)

$L H_{x, y}=O_{2 x, 2 y+1}-O_{2 x, 2 y}$

(15)

$H H_{x, y}=O_{2 x+1,2 y+1}-O_{2 x, 2 y}$

Similarly, the inverse-IWT coefficients are mathematically indicated in the Eqs. (17), (18), (19), and (20).

$O_{2 x, 2 y}=L L_{x, y}-\left|\frac{H L_{x, y}}{2}\right|$

$$
O_{2 x, 2 y+1}=L L_{x, y}+\left|\left(H L_{x, y+1}\right) / 2\right|
$$

$O_{2 x+1,2 y}=O_{2 x, 2 y+1}+L H_{x, y}-L_{x, y}$

$O_{2 x+1,2 y+1}=O_{2 x+1,2 y}+H H_{x, y}-L H_{x, y}$

Where, $1 \leq x \leq \frac{i}{2}$, and $1 \leq y \leq j / 2$ is represented as floor value, $O_{x, y}$ is specified as original foreground images, $i$ is represented as pixel height and $j$ is indicated as pixel width. The level of every foreground image pixel is denoted as $(x, y)$.

\section{D.Optimized neural network}

Similarly, optimized neural network and modified SPHIT compression techniques are applied in the background image to improve the compression ratio. Generally, the feed forward neural network utilizes the Back-Propagation (BP) algorithm as a training algorithm. Though, the BP algorithm mainly depends on the initial values of the network parameters that are usually initialized as a random value. 
In addition, the BP algorithm gets stuck with local optima that is considered as one of the major drawbacks. To compensate this problem, Genetic Algorithm (GA) and Particle Swarm Optimization (PSO) algorithm are included in feed forward neural network for strong global optimization. In an optimized neural network, GA is utilized to generate the prefect model for the PSO algorithm in which the PSO particles are guided to evolve the model. GA with diversified information of PSO particles significantly increases the search efficiency of both PSO and GA and also avoids the premature convergence of the algorithm.

In optimized neural network, the total number of iterations is equally shared by PSO and GA. Initially, the weights and bias of the neural network are chosen randomly and executes BPA until it diminishes the MSE value or the stopping criteria are satisfied. In GA, the weights and bias are represented as genes of the chromosomes. GA includes three operations such as parent selection, arithmetic crossover and mutation. In each generation, GA evaluates fitness function for all individuals in the population and replaces the worst performing individuals with best parents. Then, GA performs mutation operation in the chromosomes, if there is no improvement in the fitness function. In each iteration, the solutions provided by the GA form particles of the PSO. The GA and PSO scheme stops, if there is no further reduction in MSE or the total number of iterations is reached. The best obtained solution is stored in operation phase further compression and decompression. The parameter settings of optimized neural network are given in table 1.

Table I. Parameter settings of optimized neural network

\begin{tabular}{|c|c|}
\hline Parameters & Value \\
\hline Neural network structure & $16-4-16$ \\
\hline Block size for training & $4 \times 4$ \\
\hline Maximum no of iteration for neural network & 500 \\
\hline No of training images used for neural network & 50 \\
\hline No of testing images used for neural network & 10 \\
\hline Minimum error for GA, PSO and neural network & 0.001 \\
\hline Population size of GA and PSO & 50 \\
\hline Length of chromosomes & 148 \\
\hline Inertia factor of PSO & 1 \\
\hline No of iteration & 100 \\
\hline Crossover function & 0.8 \\
\hline Mutation function & 0.1 \\
\hline Best acceleration factor of PSO & 1.5 and 2.0 \\
\hline Lower and upper bound variable & {$[-55]$} \\
\hline
\end{tabular}

\section{E.Modified SPIHT algorithm}

In the modified SPIHT algorithm, each state is determined by means of bit plane, which is quantified by hierarchical tree structure. The spatial orientation tree coefficients are coded from the most significant bit plane to the least significant bit plane. In every bit-plane, the SPIHT algorithm determines the threshold value on the basis of the following three steps that are detailed below.

1st step: Initially encode the list of insignificant image pixels whose magnitudes are higher than the threshold value. This step tracks the image pixels, which need to be calculated.

2nd step: In the next step, encode the list of insignificant sets that contain wavelet coefficients, which are determined by a tree structure. Compared to the threshold value (insignificant), the encoded wavelet coefficients have smaller magnitudes. Generally, the list of insignificant sets excluded the tree-coefficients and all sub-tree roots that have at least four elements. Here, the sub-tree roots are not excluded, because it quantizes and encodes the wavelet coefficients effectively that delivers excellent rate distortion properties in the noise free environment. These wavelet coefficients deliver more information to the image edges and its vicinity improves the subjective visual experience.

3rd step: In the list of significant pixels, the refinement bit of the $\left(n^{\text {th }}\right)$ coefficients have higher magnitudes than the threshold value. This step tracks the image pixels that are all already calculated.

\section{EXPERIMENTAL OUTCOME AND DISCUSSION}

In this paper, the proposed progressive image transmission system was simulated by using MATLAB (version 2018a). In order to validate the efficiency of proposed transmission system, the performance of the proposed progressive image transmission system was compared with a previous research work (SPIHT with embedded zerotree wavelets [15]). In this article, the proposed transmission system performance was validated in light of MSE, PSNR, SSIM, and compression ratio.

\section{A.Performance metric}

Performance metric is defined as the procedure of analysing and collecting information about the performance of an individual or group. The relationship between the input and output variables of the proposed progressive image transmission system is understood by utilizing the performance measures: MSE, PSNR, SSIM, and compression ratio. The mathematical formulas of MSE, PSNR, SSIM, and compression ratio are denoted in Eqs. (21), (22), (23), and (24).

$M S E=1 / i j \Sigma_{p=0}^{i-1} \Sigma_{q=0}^{j-1}[I(x, y)-k(x, y)]^{2}$

$P S N R=10 \log _{10}\left(\frac{255^{2}}{M S E}\right)$

Where, $I(x, y)$ is specified as compressed image, $k(x, y)$ is denoted as uncompressed image, $i$ and $j$ are indicated as height and width of the image.

$\operatorname{SSIM}(x, y)=\frac{\left(2 \mu_{x} \mu_{y}+c_{1}\right)\left(2 \sigma_{x y}+c_{2}\right)}{\left(\mu_{x}^{2}+\mu_{y}^{2}+c_{1}\right)\left(\sigma_{x}^{2}+\sigma_{y}^{2}+c_{2}\right)}$

Where, $x$ and $y$ are indicated as windows of compressed image $I$ and uncompressed image $k, \sigma$ and $\mu$ are signified as standard deviation and mean of $x$ and $y, c_{1}$ and $c_{2}$ are denoted as constants. The image compression ratio is determined as the ratio between compressed and uncompressed image size.

Published By:

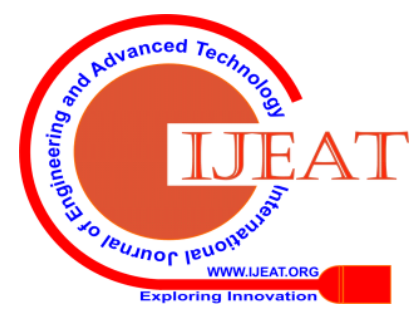


An Effective Progressive Image Transmission using Superpixel based Saliency Detection and Modified SPIHT Compression Algorithm

Compression ratio $=$

$$
\frac{\text { Uncompressed image size }}{\text { Compressed image size }}
$$

\section{B. Quantitative research on wildlife images}

In this segment, wildlife images are utilized to assess the performance of the proposed progressive image transmission system. During image transmission, the foreground region adopts lossless compression transmission and the background region adopts lossy compression transmission for enhancing the efficiency of image transmission. In table
2, the average PSNR and compression ratio of saliency region and full image are $98.6904 \mathrm{~dB}, 43.32$ and $44.15 \mathrm{~dB}$, 81.04. From the experimental simulation, the proposed progressive image transmission system ensures the reconstruction quality of the saliency object region and full image. Fig. 4 states the progressive transmission effect of wildlife images. Graphical comparison of reconstructed wildlife images in light of compression ratio and PSNR is denoted in Fig. 5.
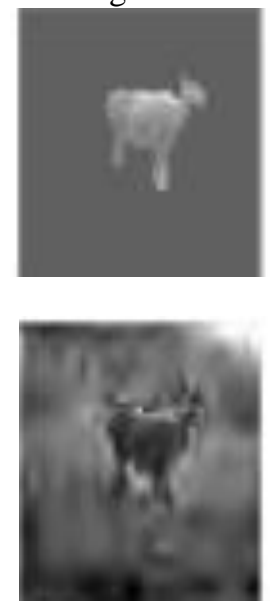
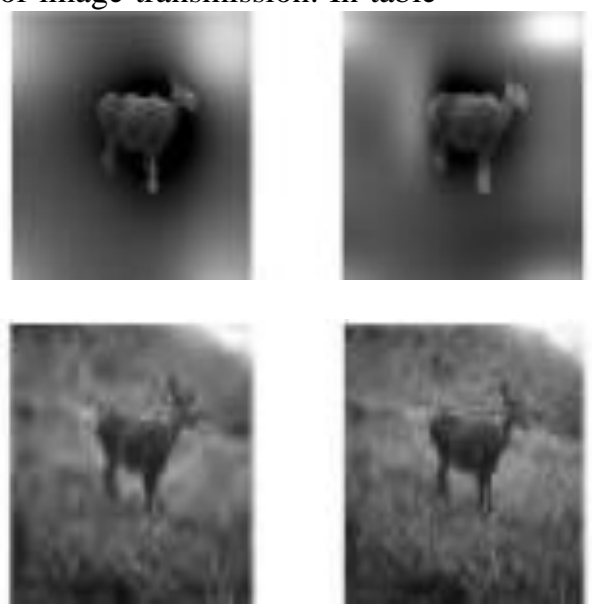

Fig. 4. Progressive transmission effect of wildlife images
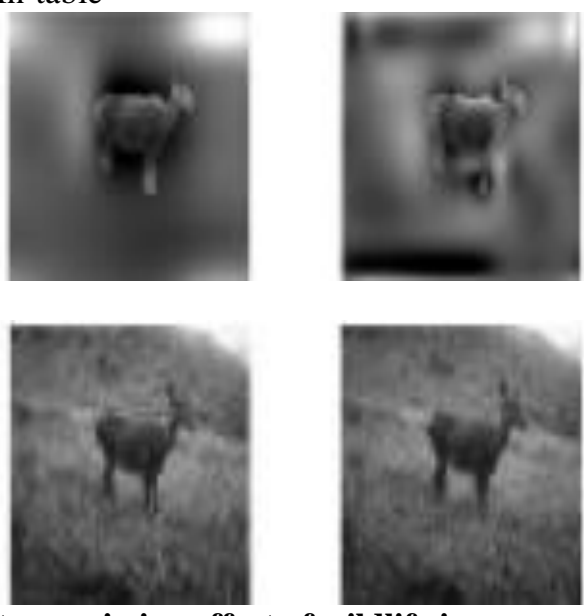

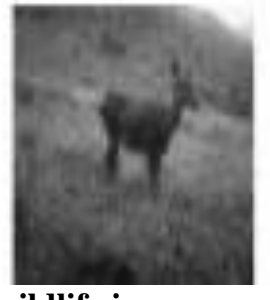

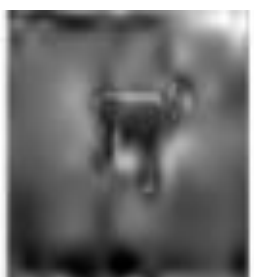

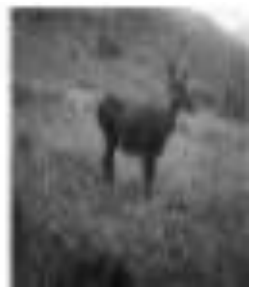

Table II. Effect of reconstructed wildlife images in light of PSNR and compression ratio

\begin{tabular}{|c|c|c|c|c|}
\hline \multicolumn{5}{|c|}{ Wildlife images } \\
\hline \multirow[b]{2}{*}{$\begin{array}{c}\text { Sample } \\
\text { No }\end{array}$} & \multicolumn{2}{|c|}{ PSNR (dB) } & \multicolumn{2}{|c|}{$\begin{array}{c}\text { Compression } \\
\text { ratio }\end{array}$} \\
\hline & $\begin{array}{c}\text { Saliency } \\
\text { region }\end{array}$ & $\begin{array}{c}\text { Full } \\
\text { image }\end{array}$ & $\begin{array}{c}\text { Saliency } \\
\text { region }\end{array}$ & $\begin{array}{c}\text { Full } \\
\text { image }\end{array}$ \\
\hline 1 & 98.492 & 40.965 & 44.21 & 85.21 \\
\hline 2 & 98.58 & 48.969 & 42.7 & 81.23 \\
\hline 3 & 98.72 & 41.308 & 32.3 & 79.23 \\
\hline 4 & 98.63 & 48.17 & 54.21 & 75.66 \\
\hline 5 & 99.03 & 41.338 & 43.18 & 83.87 \\
\hline Mean & 98.6904 & 44.15 & 43.32 & 81.04 \\
\hline
\end{tabular}

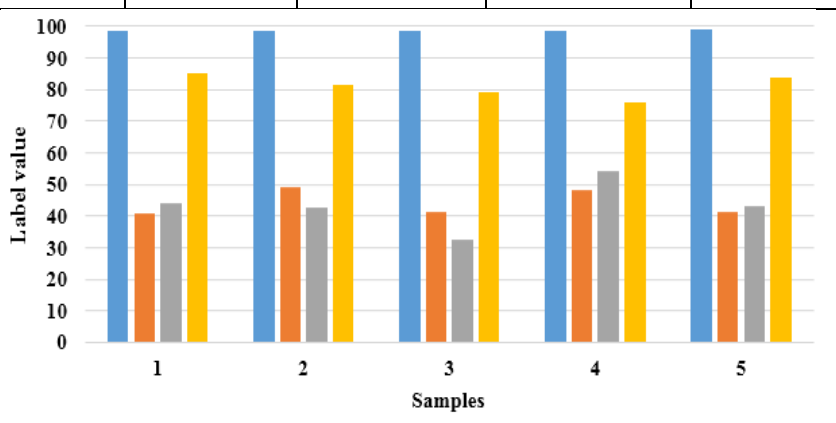

॥SNR Saliency region $\quad$ PSNR Full image

$\square$ Compression ratio Saliency region $₫$ Compression ratio Full image

Fig. 5. Graphical comparison of reconstructed wildlife images in light of compression ratio and PSNR

Similarly, in table 3, the average SSIM and MSE value of saliency region and full image are 0.984, 0.00000879 and
0.92492, 3.320266. In the transmission procedure, reconstructed wildlife image information is used to recognize the species of wildlife and then the transmission process is completed, once the satisfactory information is achieved.

Table III. Effect of reconstructed wildlife images in light of SSIM and MSE

\begin{tabular}{|c|c|c|c|c|}
\hline \multicolumn{5}{|c|}{ Wildlife images } \\
\hline \multirow{2}{*}{$\begin{array}{c}\text { Samp } \\
\text { le No }\end{array}$} & $\begin{array}{c}\text { Saliency } \\
\text { region }\end{array}$ & $\begin{array}{c}\text { Full } \\
\text { image }\end{array}$ & $\begin{array}{c}\text { Saliency } \\
\text { region }\end{array}$ & $\begin{array}{c}\text { Full } \\
\text { image }\end{array}$ \\
\hline 1 & 0.99 & 0.94556 & 0.00000920 & 5.2068 \\
\hline 2 & 0.98 & 0.97744 & 0.00000900 & 0.82442 \\
\hline 3 & 0.99 & 0.95917 & 0.00000872 & 4.811 \\
\hline 4 & 0.97 & 0.85963 & 0.00000891 & 0.98911 \\
\hline 5 & 0.99 & 0.8828 & 0.00000812 & 4.77 \\
\hline Mean & 0.984 & 0.92492 & 0.00000879 & 3.320266 \\
\hline
\end{tabular}

\section{C.Quantitative research on medical images}

In this segment, the medical images with high noise interference, high resolution, and complex background are selected from the DICOM dataset for image transmission. In the proposed transmission system, initially the saliency region is transmitted through a lossless method, and then the background region in a lossy way, which is specified in Fig. 1. The progressive transmission effect of medical images is gra30phically denoted in Fig. 6. In table 4, the average compression ratio and PSNR of saliency region and full image are 46.28, $98.9472 \mathrm{~dB}$, and 86.88, $40.828 \mathrm{~dB}$, respectively. Graphical comparison of reconstructed medical images in light of the compression ratio and PSNR is represented in Fig. 7.

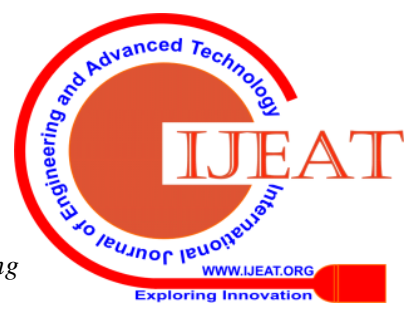



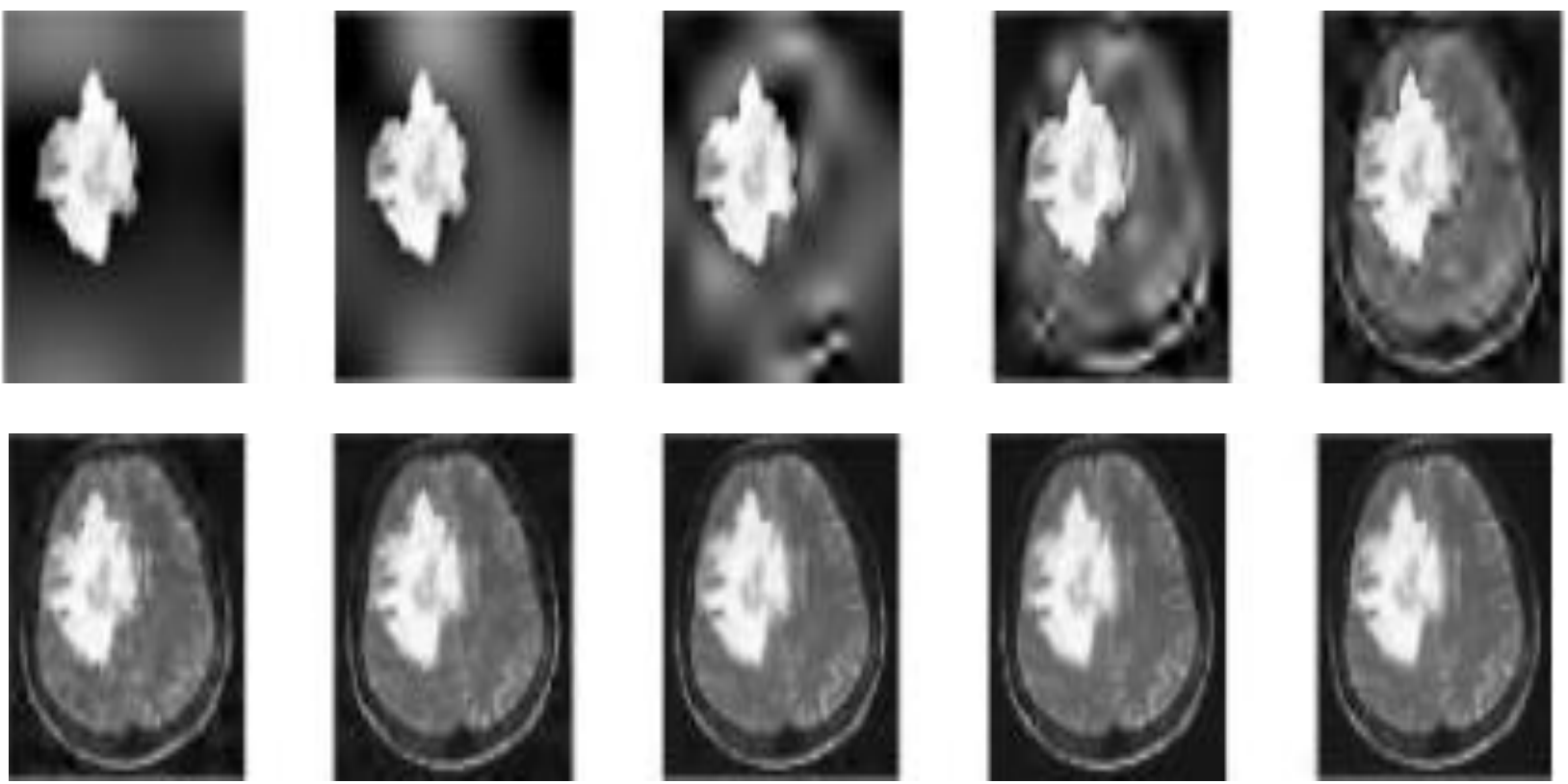

Fig. 6. Progressive transmission effect of medical images

Table IV. Comparison effect of reconstructed medical images in light of PSNR and compression ratio

\begin{tabular}{|c|c|c|c|c|}
\hline \multicolumn{5}{|c|}{ Medical images } \\
\hline \multirow[b]{2}{*}{$\begin{array}{c}\text { Sample } \\
\text { No }\end{array}$} & \multicolumn{2}{|c|}{ PSNR (dB) } & \multicolumn{2}{|c|}{ Compression ratio } \\
\hline & $\begin{array}{c}\text { Saliency } \\
\text { region }\end{array}$ & $\begin{array}{c}\text { Full } \\
\text { image }\end{array}$ & $\begin{array}{l}\text { Saliency } \\
\text { region }\end{array}$ & $\begin{array}{c}\text { Full } \\
\text { image }\end{array}$ \\
\hline 1 & 98.258 & 44.38 & 42.17 & 86.17 \\
\hline 2 & 98.730 & 41.62 & 41.14 & 88.14 \\
\hline 3 & 99.534 & 35.44 & 44.28 & 89.28 \\
\hline 4 & 99.624 & 41.60 & 52.16 & 87.16 \\
\hline 5 & 98.59 & 41.10 & 51.65 & 83.65 \\
\hline Mean & 98.9472 & 40.828 & 46.28 & 86.88 \\
\hline
\end{tabular}

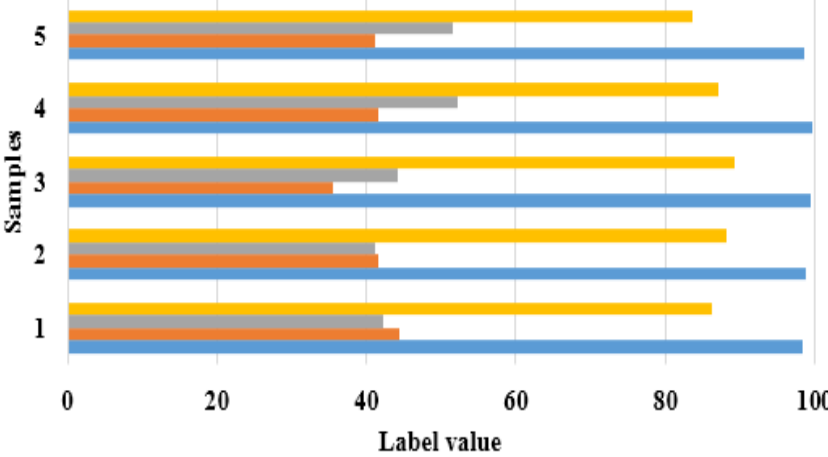

- Compression ratio Full image $₫$ Compression ratio Saliency region - PSNR Full image

- PSNR Saliency region

Fig. 7. Graphical comparison of reconstructed medical images in light of compression ratio and PSNR

Table V. Comparison effect of reconstructed medical images in light of SSIM and MSE

\begin{tabular}{|c|c|c|c|c|}
\hline \multicolumn{5}{|c|}{ Medical images } \\
\hline \multirow{2}{*}{$\begin{array}{c}\text { Sample } \\
\text { No }\end{array}$} & $\begin{array}{c}\text { SSIM } \\
\text { Saliency } \\
\text { region }\end{array}$ & $\begin{array}{c}\text { Full } \\
\text { image }\end{array}$ & Saliency region & $\begin{array}{c}\text { Full } \\
\text { image }\end{array}$ \\
\hline 1 & 0.99 & 0.8215 & 0.00000971 & 2.369 \\
\hline 2 & 0.98 & 0.8544 & 0.00000871 & 4.474 \\
\hline 3 & 0.99 & 0.70 & 0.00000724 & 18.56 \\
\hline 4 & 0.98 & 0.74 & 0.00000709 & 4.49 \\
\hline
\end{tabular}

\begin{tabular}{|c|c|c|c|c|}
\hline 5 & 0.99 & 0.73 & 0.00000899 & 5.04 \\
\hline Mean & 0.986 & 0.76918 & 0.000008348 & 6.9866 \\
\hline
\end{tabular}

Similarly, in table 5, the average SSIM and MSE of saliency region and full image are 0.986, 0.000008348, and 0.76918, 6.9866. In the transmission procedure, reconstructed image information is utilized for recognizing the tumor region and then terminate the transmission after achieving the satisfactory information.

\section{D.Comparative analysis}

Table 6 denotes the comparative study of proposed and existing work in light of PSNR value. W. Feng et al. [15] presented a new progressive transmission system to transmit the saliency object and background region with different coding priorities and strategies. In this existing work, convolutional neural network was used for detecting the saliency object region.

Table VI. Comparative study of existing and proposed work by means of PSNR

\begin{tabular}{|c|c|c|c|c|}
\hline \multicolumn{4}{|c|}{ PSNR (dB) } \\
\hline \multirow{2}{*}{$\begin{array}{c}\text { Sample } \\
\text { No }\end{array}$} & \multicolumn{2}{|c|}{ Proposed } & \multicolumn{2}{c|}{ Existing [15] } \\
\cline { 2 - 5 } & Saliency & $\begin{array}{c}\text { Full } \\
\text { region }\end{array}$ & $\begin{array}{c}\text { Saliency } \\
\text { region }\end{array}$ & $\begin{array}{c}\text { Full } \\
\text { image }\end{array}$ \\
\hline 1 & 98.492 & 40.965 & 45.43 & 41.47 \\
\hline 2 & 98.58 & 48.969 & 47.16 & 39.28 \\
\hline 3 & 98.72 & 41.308 & 45.34 & 37.51 \\
\hline 4 & 98.63 & 48.17 & 46.65 & 41.18 \\
\hline 5 & 99.03 & 41.338 & 44.30 & 36.90 \\
\hline Mean & 98.6904 & 44.15 & 45.776 & 39.268 \\
\hline
\end{tabular}

Additionally, SPIHT coding was applied to transmit the saliency images and the background region was transmitted using embedded zerotree wavelets. This research work performance was validated on wildlife images. From the experimental outcome, the existing work achieved average PSNR value of saliency region and full image were 45.776 $\mathrm{dB}$ and $39.268 \mathrm{~dB}$.

Published By:

Blue Eyes Intelligence Engineering \& Sciences Publication

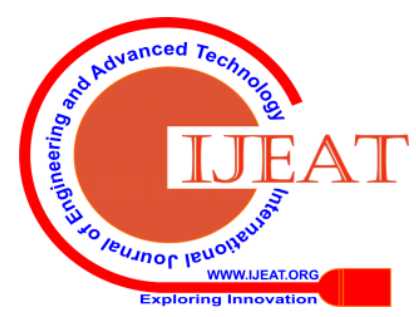


Related to this existing work, the proposed progressive image transmission system achieved better performance, because it effectively preserves the edge information of the images.

\section{CONCLUSION}

A new progressive image transmission system is proposed in this paper for both color and graylevel images on the basis of superpixel based saliency detection and modified SPIHT algorithm. The key benefit of the proposed transmission system is that it delivers a good quality of images at the early stages with low bit rate that saves the channel cost in case of premature termination. The proposed work performance represents its efficacy and suitability in the field of multimedia search. Related to the existing work (SPIHT with embedded zerotree wavelets), the proposed work delivered an effective performance in light of PSNR, SSIM, compression ratio, and MSE. From this research study, the proposed work averagely attained $44.15 \mathrm{~dB}$ of PSNR value in wild life images that is superior related to the previous research work. In future work, a new compression algorithm is combined with optimized neural network for further enhancing the performance of progressive image transmission.

\section{ACKNOWLEDGEMENT}

The authors would like to thank Doctoral committee members Dr.Raviraj P, Professor, GSSSITW, Mysuru and Dr. Shreekanth T, Project Manager, L\&T Technology Services, Mysuru and also Dr. Shashikiran.B.R, Consultant radiologist, Sri Sai ultrasound centre, Hassan for their comments and suggestions in order to make this research work better.

\section{REFERENCES}

1. H. C. Huang, Y. Y. Lu, and J. Lin. (2016). Ownership protection for progressive image transmission with reversible data hiding and visual secret sharing. Optik. 127. pp. 5950-5960.

2. B. C. Dhara, and B. Chanda. (2012). A fast progressive image transmission scheme using block truncation coding by pattern fitting. Journal of Visual Communication and Image Representation. 23. pp. 313-322.

3. C. C. Chang, Y. C. Li, and C. H. Lin. (2008). A novel method for progressive image transmission using blocked wavelets. AEU-International Journal of Electronics and Communications. 62. pp.159-162.

4. M. Amiri, H. Danyali, B. Zahir-Azami, and F. Tab. (2013). Adaptive, scalable and robust watermarking for wavelet-based progressive image transmission. The Imaging Science Journal. 61. pp. 120-133.

5. J. Villanueva-Oller, R. J. Villanueva, and S. Díez. (2007). CASANDRA: A prototype implementation of a system of network progressive transmission of medical digital images. computer methods and programs in biomedicine. 85. pp. 152-164.

6. Y. C. Lin. (2011). Reversible data-hiding for progressive image transmission. Signal Processing: Image Communication. 26. pp. 628-645.

7. S. S. Arslan, P. C. Cosman, and L. B. Milstein. (2011). Coded hierarchical modulation for wireless progressive image transmission. IEEE Transactions on Vehicular technology. 60(9). pp. 4299-4313.

8. N. K. Lim, D.Y. Kim, and H. Lee. (2010). Interactive progressive image transmission for realtime applications. IEEE Transactions on Consumer Electronics. 56(4). pp. 2438-2444.

9. E. M. Rubino, D. Centelles, J. Sales, J. V. Marti, R. Marin, P. J. Sanz, and A. J. Alvares. (2017). Progressive image compression and transmission with region of interest in underwater robotics. In OCEANS 2017-Aberdeen. pp. 1-9, 2017.
10. P. Peter, C. Schmaltz, N. Mach, M. Mainberger, and J. Weickert. (2015) Beyond pure quality: Progressive modes, region of interest coding, and real time video decoding for PDE-based image compression. Journal of Visual Communication and Image Representation. 31. pp. 253-265.

11. L. Liu, A. Wang, C. C. Chang, and Z. Li. (2014). A novel real-time and progressive secret image sharing with flexible shadows based on compressive sensing. Signal Processing: Image Communication. 29(1). pp. 128-134.

12. W. Feng, J. Zhang, C. Hu, Y. Wang, Q. Xiang, and H. Yan. (2018). A novel saliency detection method for wild animal monitoring images with WMSN. Journal of Sensors.

13. N. Jiang, Y. Zhuang, and D. K. Chiu. (2017). Multiple transmission optimization of medical images in recourse-constraint mobile telemedicine systems. Computer methods and programs in biomedicine, 145. pp. 103-113.

14. H. Kim, R. Annavajjala, P. C. Cosman, and L. B. Milstein. (2010). Source-channel rate optimization for progressive image transmission over block fading relay channels. IEEE Transactions on Communications. 58(6). pp. 1631-1642.

15. W. Feng, C. Hu, Y. Wang, J. Zhang, and H. Yan. A Novel Hierarchical Coding Progressive Transmission Method for WMSN Wildlife Images. Sensors. 19(4). pp. 946, 2019.

16. E. M. Rubino, D. Centelles, J. Sales, J. V. Martí, R. Marín, P. J. Sanz, and A. J. Alvares. Underwater radio frequency image sensor using progressive image compression and region of interest. Journal of the Brazilian Society of Mechanical Sciences and Engineering. 39(10). pp. 4115-4134.

17. Y. Fang, X. Zhang, and N. Imamoglu. (2018). A novel superpixel-based saliency detection model for 360-degree images. Signal Processing: Image Communication. 69. pp. 1-7.

18. T. Venugopal, and V. S. K. Reddy. (2018). Image Watermarking Using Two Level Encryption Method Based on Chaotic Logistic Mapping and Rivest Shamir Adleman Algorithm. International Journal of Intelligent Engineering and Systems. 11(6). pp. 271-281.

19. https://www.kaggle.com/navoneel/brain-mri-images-for-brain-tumor-d etection

\section{AUTHORS PROFILE}

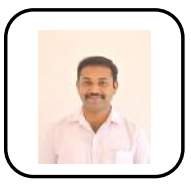

Ravikiran H.K. received the B.E. degree in Electronics and Communication Engineering from S.J.C. Institute of Technology, Chikballapur, Karnataka, India in the year 2008, and M.Tech. in Digital Electronics and Communication Systems from Malnad College of Engineering, Hassan, Karnataka, India, in 2010. He has a Teaching experience of 9 years. He has published 7 papers till date, of which 5 papers are from International Journals, rest are from conference. His subjects of interest include Image Processing, Soft computing techniques etc.

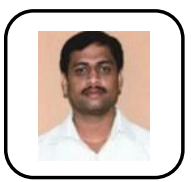

Dr. Jayanth J. received the B.E. degree in Electronics and Communication Engineering from Vidya Vikas Institute of Engineering and Technology, Mysore, Karnataka, India in the year 2008, and M.Tech. in Digital Electronics and Communication Systems from Malnad College of Engineering, Hassan, Karnataka, India, in 2010 and Ph.D from VTU, Karnataka, India, in 2017. He has a Teaching experience of 9 years. He has published 35 papers till date, of which 15 papers are from International Journals, rest are from conference. He has authored 3 Book chapter and written 3 books on Image Fusion and Image classification using fuzzy logic for remote sensed data classification. He is working as a PI for the DST NRDMS project research grant. He has completed 3 consultancy projects for Infy-cloud Bangalore. His subjects of interest include Image Processing, Swarm Intelligence and Remote Sensing. 